\title{
Elucidation of kinematical and dynamical structure of the Galactic bulge
}

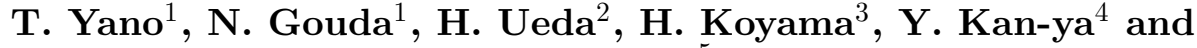 \\ A. Taruya ${ }^{5}$ \\ ${ }^{1}$ National Astronomical Observatory, Mitaka, Tokyo 181-8588, Japan \\ email: yano.t@nao.ac.jp, naoteru.gouda@nao.ac.jp \\ ${ }^{2}$ Faculty of Education and Human Studies, Akita University, Tegata-gakuen, \\ Akita 010-0852, Japan \\ email: ueda@ed.akita-u.ac.jp \\ ${ }^{3}$ Department of Physics, Nagoya University, Chikusa-ku, Nagoya 464-8602, Japan \\ email: koyama.hiroko@gmail.com \\ ${ }^{4}$ Department of Astronomy, Yonsei University 134 Shinchong-dong, Seodaemun-gu, Seoul \\ 120-749, South Korea \\ email: ykanya@galaxy.yonsei.ac.kr \\ ${ }^{5}$ School of Science, The University of Tokyo, Tokyo 113-0033, Japan \\ email: ataruya@utap.phys.s.u-tokyo.ac.jp
}

\begin{abstract}
Future space mission of astrometric satellite, GAIA and JASMINE (Japan Astrometry Satellite Mission for Infrared Exploration), will produce astrometric parameter, such as positions, parallaxes, and proper motions of stars in the Galactic bulge. Then kinematical information will be obtained in the future. Accordingly it is expected that our understanding of the dynamical structure will be greatly improved. Therefore it is important to make a method to construct a kinematical and dynamical structure of the Galactic bulge immediately.
\end{abstract}

Keywords. Galaxy: bulge, Galaxy: kinematics and dynamics, astrometry, methods: analytical, methods: data analysis

\section{Overview on the Construction of Dynamical Structure of the Galactic Bulge}

Here we outline the method to obtain dynamical structure of the Galactic bulge (see Fig. 1).

1. We assume a gravitational potential, $V(q)$, and a distribution function (hereafter $\mathrm{DF}), f_{\text {model }}(J)$. Both must be assumed in consistent with Poisson equation.

2. We obtain $J(p, q)$, the relation between $J$ and $(p, q)$, numerically from the gravitational potential using torus fitting method.

3. We obtain $f_{\text {model }}(J(p, q))$ from $J(p, q)$ and assumed DF, $f_{\text {model }}(J)$.

4. We estimate the observed DF, $f_{C \text { model }}(p, q)$, from the modeled DF, $f_{\text {model }}(J(p, q))$, by the convolution procedure.

5. We compare $f_{C m o d e l}(J(p, q))$ with $f_{\text {obs }}(p, q)$. Or equivalently we can compare $f_{\text {model }}(J(p, q))$ with deconvolved DF, $f_{\text {Dobs }}(p, q)$.

\section{Torus Fitting Method}

This method is based on the splendid idea shown by McGill \& Binney(1990), Kaasalainen \& Binney (1993), and so on. These works are to obtain torus of a given potential and $J(p, q)$ as a function of momenta and positions numerically. However, it is very difficult 


\section{Flow Chart for constructing dynamical structure of the Galactic bulge}

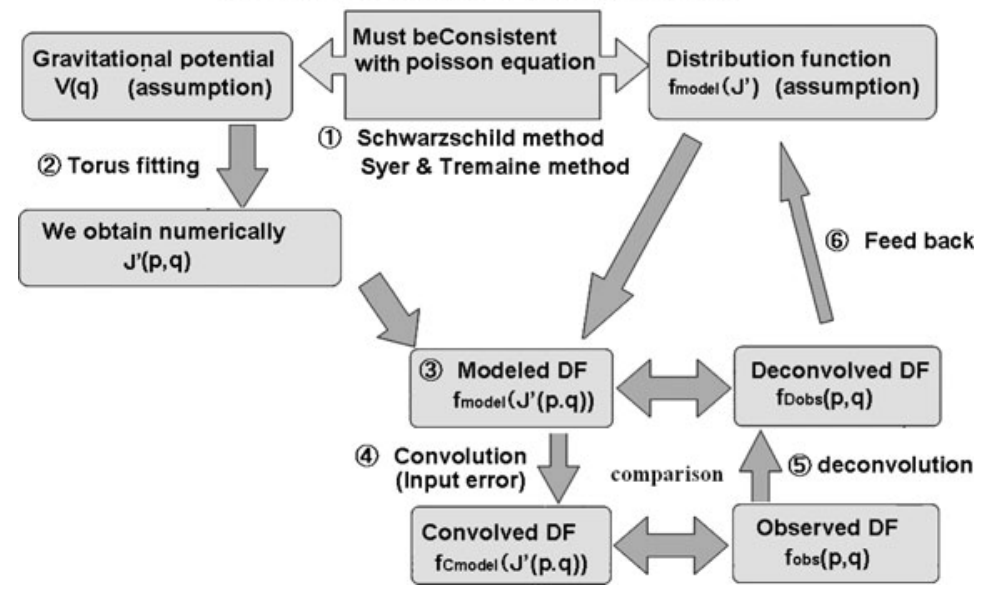

Figure 1. Flow chart for constructing dynamical structure of the Galactic bulge.

to construct torus and $J(p, q)$ without complicated procedure except for one dimensional case. Because they do not utilize any geometrical information of the torus. Then we concentrate our attention on getting the coordinate transformation between the action variables, $J$, and the Cartesian coordinate, $(x, p)$. Accordingly we utilize directly the information of the shape of torus by calculated numerically in advance. The procedure is as follows:

1. Prepare action variables $J^{\prime}$.

2. Target torus is calculated with given $J^{\prime}$, and a type of Hamiltonian is determined (harmonic oscillator or isochrone potential).

3. Prepare Np sets $\left(J^{\prime}, q_{i}\right)$ with $i=1,2,3, \cdots, N p$.

4. Evaluate $\left(J, q_{i}\right)$ from the geometrical information of the target torus.

5. Revise $J$ to satisfy equation $(2.1)$.

6. Iterate (4)-(6) and evaluate $J_{i}$.

7. Determine $S_{n}$ from equation (2.1).

$$
J=J^{\prime}+\sum n S_{n} \cos n q
$$

\section{Future Work}

We must costruct a gravitational potential, $V(q)$, and a DF consistently. The Schwarzschild method and Syer \& Tremaine (1996) method can be powerful tools.

After we make a model of DF, we must compare the DF with the observational data. We consider quantitatively the relation between the number of the observed stars and the acculacy of the estimation of the DF.

\section{References}

McGill, C. \& Binney, J. J. 1990, MNRAS 244, 634

Kaasalainen, M. \& Binney, J. J. 1994, MNRAS 268, 1033

Syer, D. \& Tremaine, S. 1996, MNRAS 282, 223 


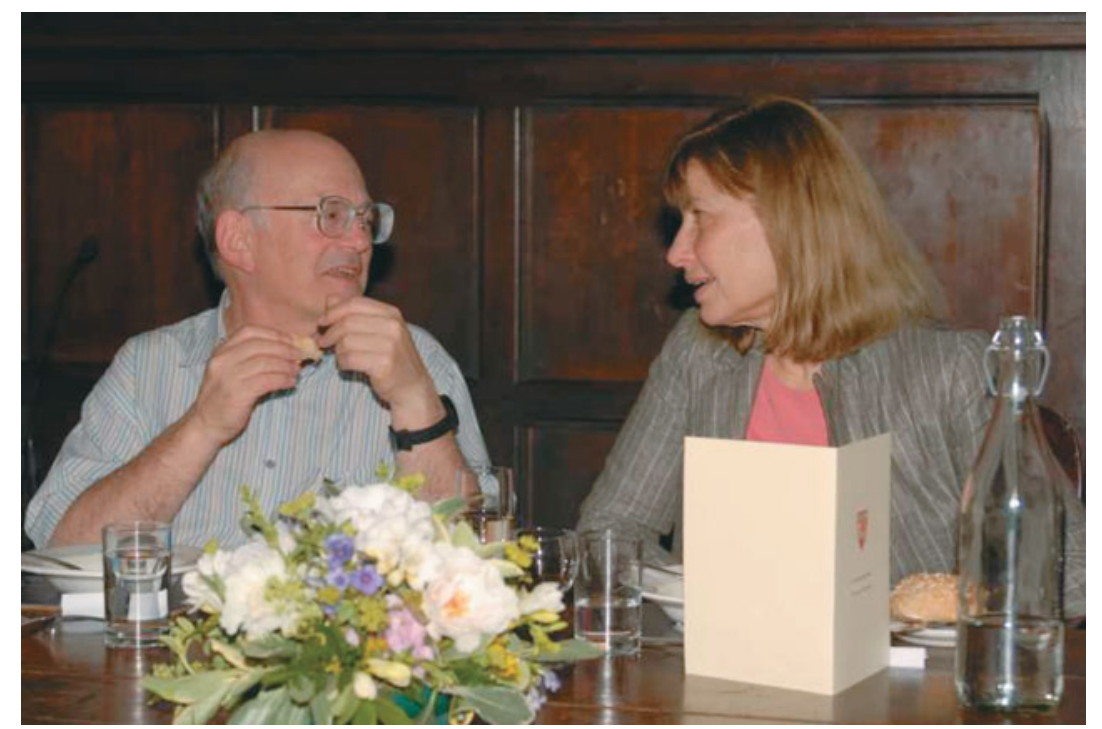

Figure 1. SOC members Ken Freeman and Beatriz Barbuy at high table during the symposium dinner.

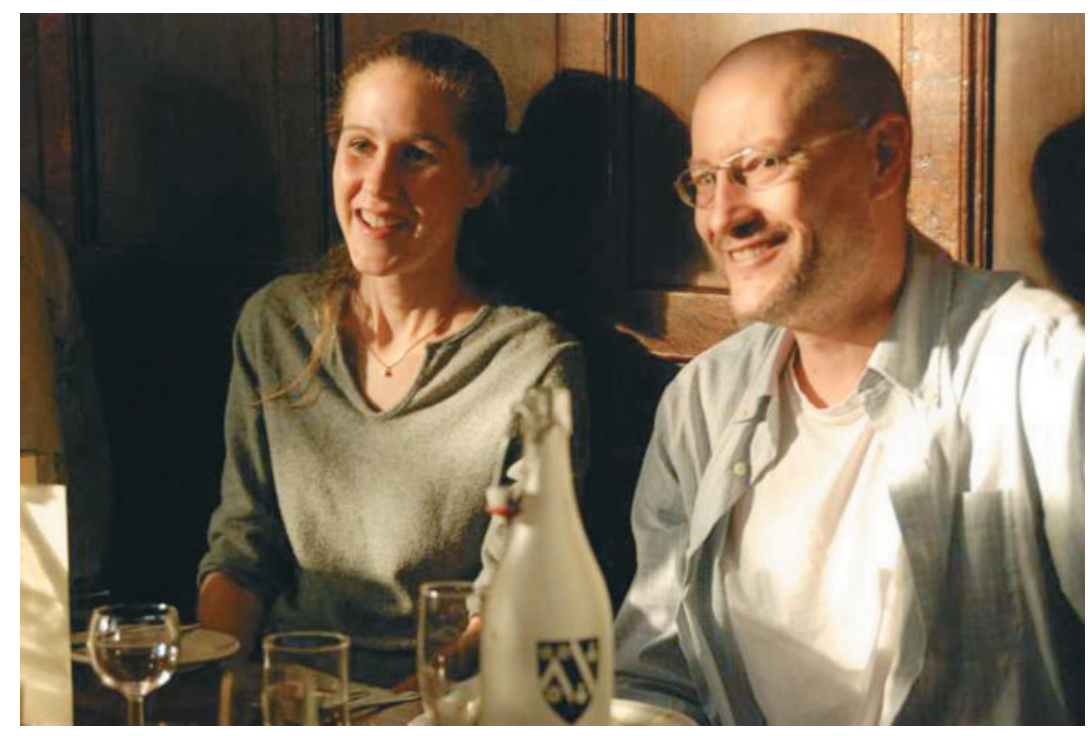

Figure 2. Lauren MacArthur and Brad Gibson during the symposium dinner. 


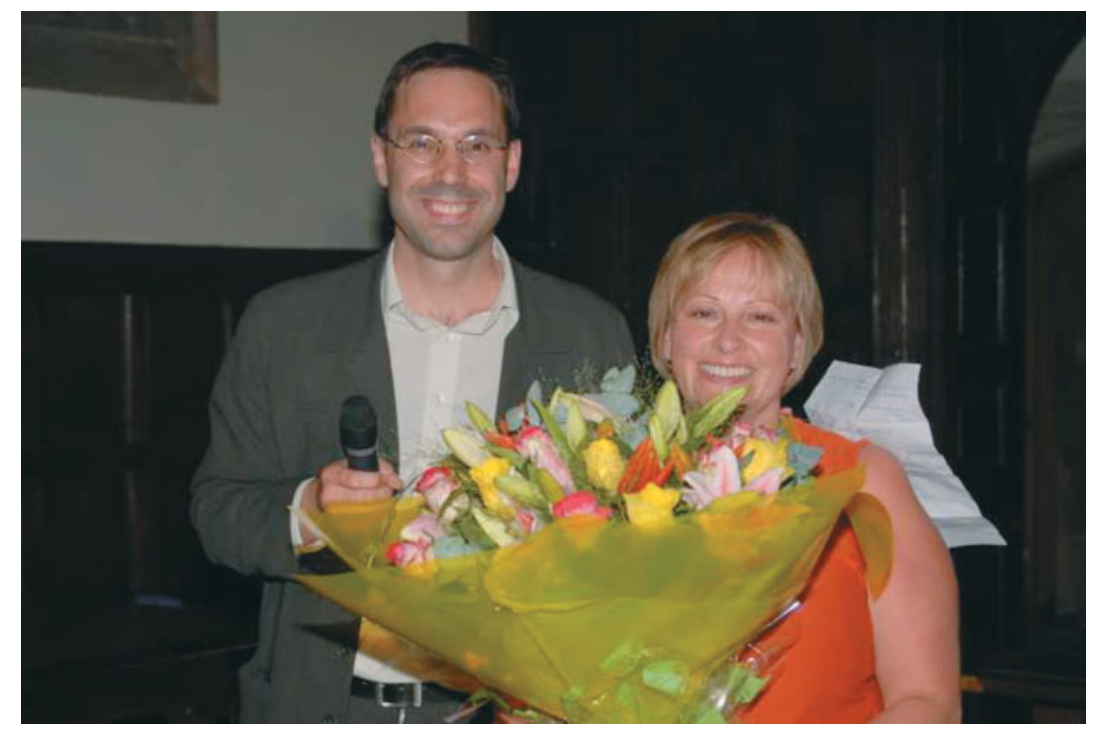

Figure 3. SOC and LOC chair Martin Bureau thanking symposium assistant Vanessa Ferraro-Wood for her hard work.

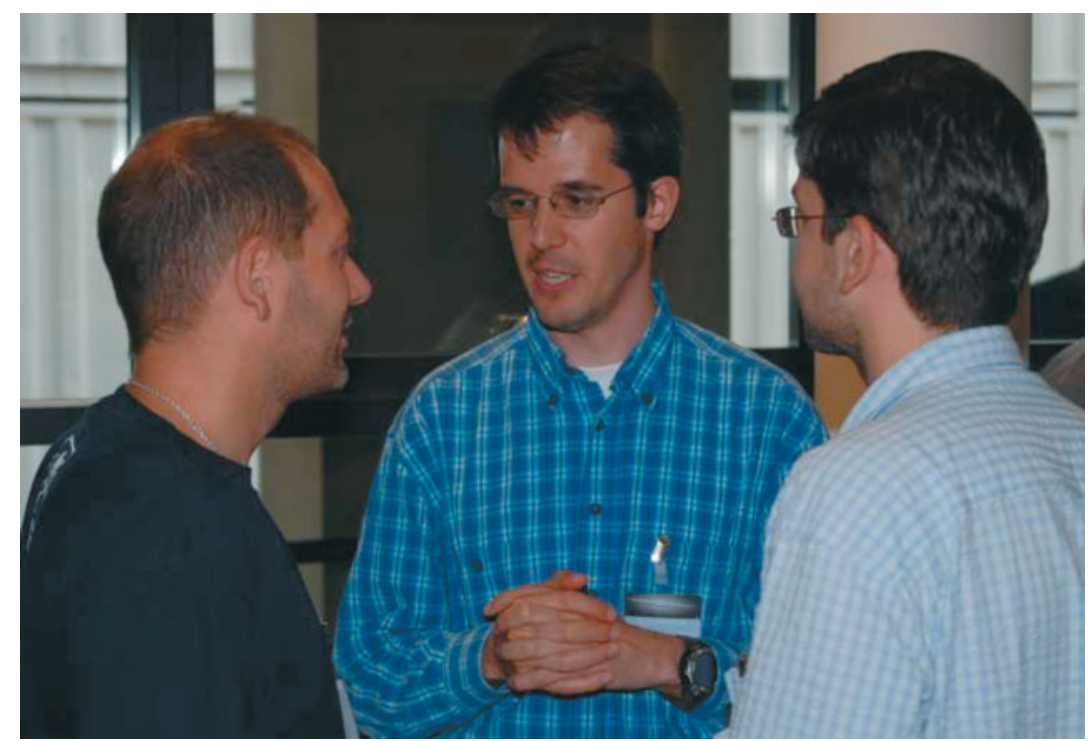

Figure 4. Eric Emsellem, Michele Cappellari and LOC member Davor Krajnović discussing during a coffee break. 


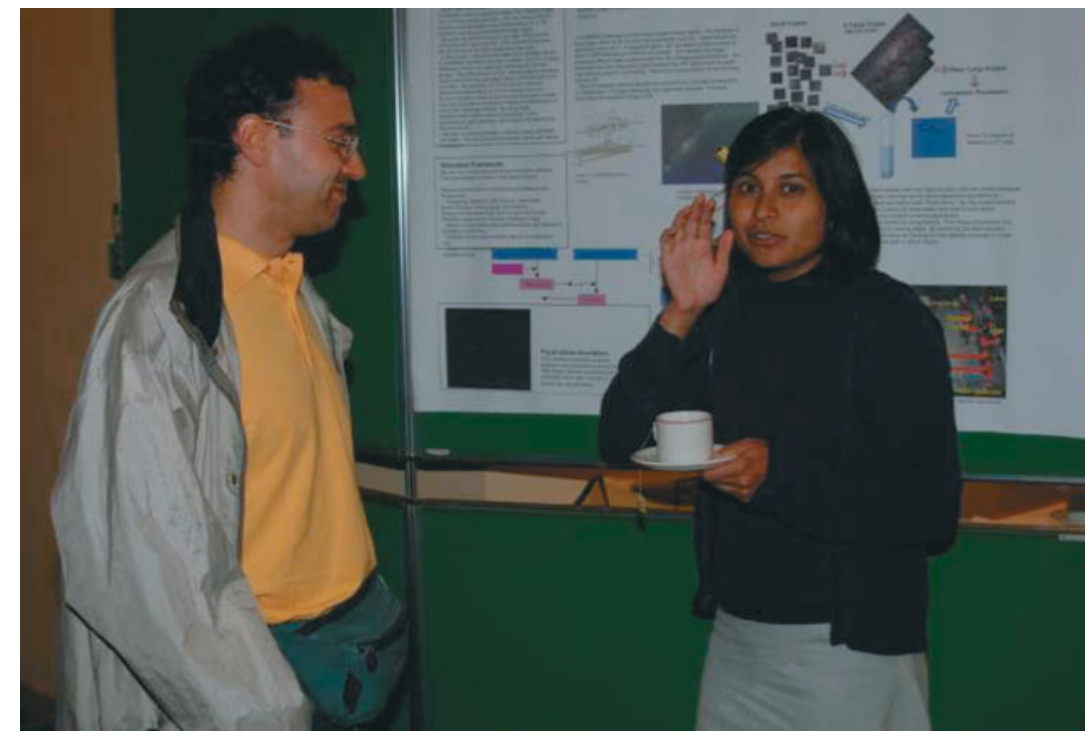

Figure 5. Luca Baiotti and Samaya Nissanke discussing in front of a poster.

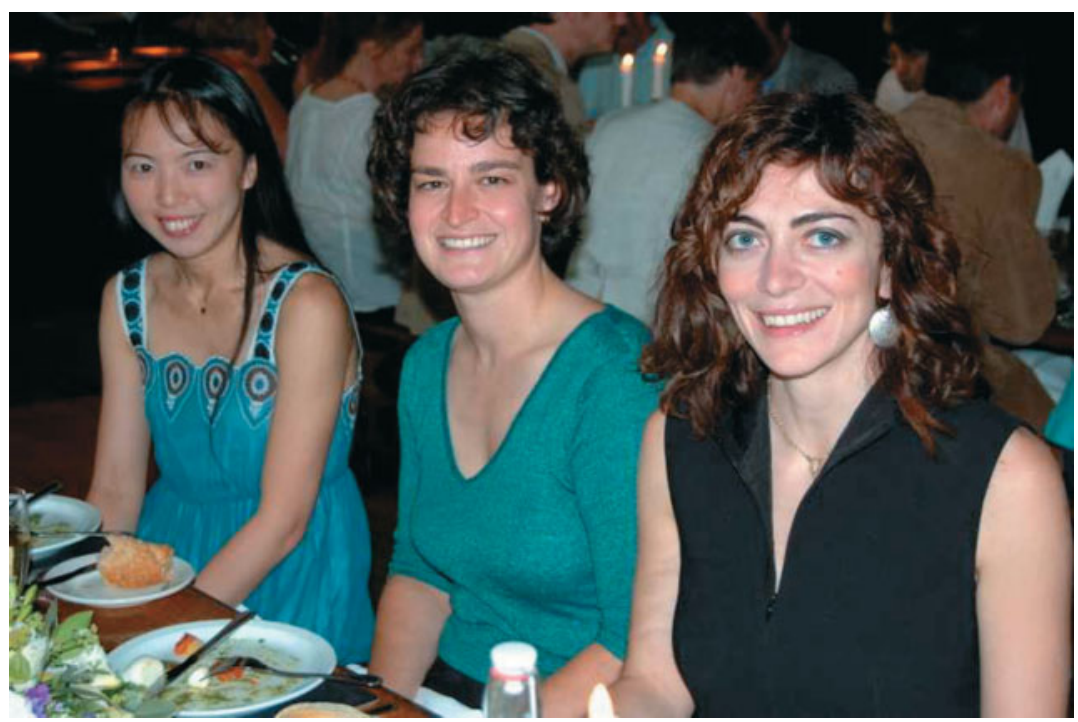

Figure 6. Chiaki Kobayashi, Genevieve Graves and Elena D'Onghia during the symposium dinner. 


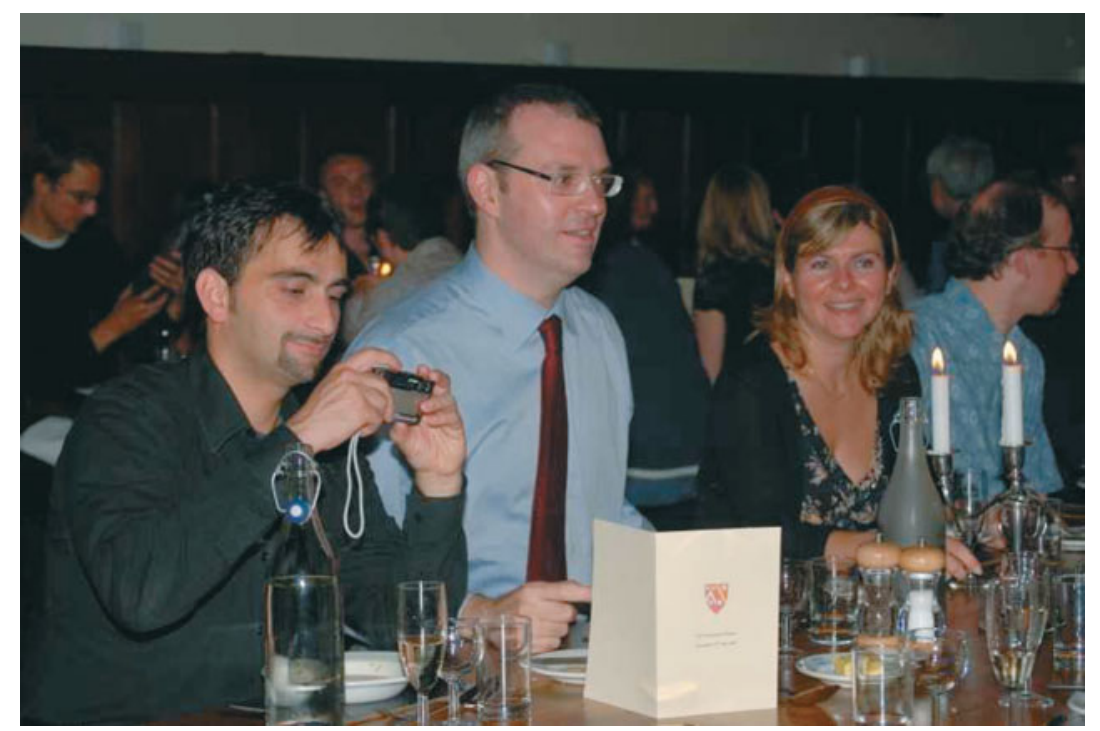

Figure 7. LOC member Marc Sarzi, Enrico Corsini, Antonella Vallenari and Bryan Miller during the symposium dinner.

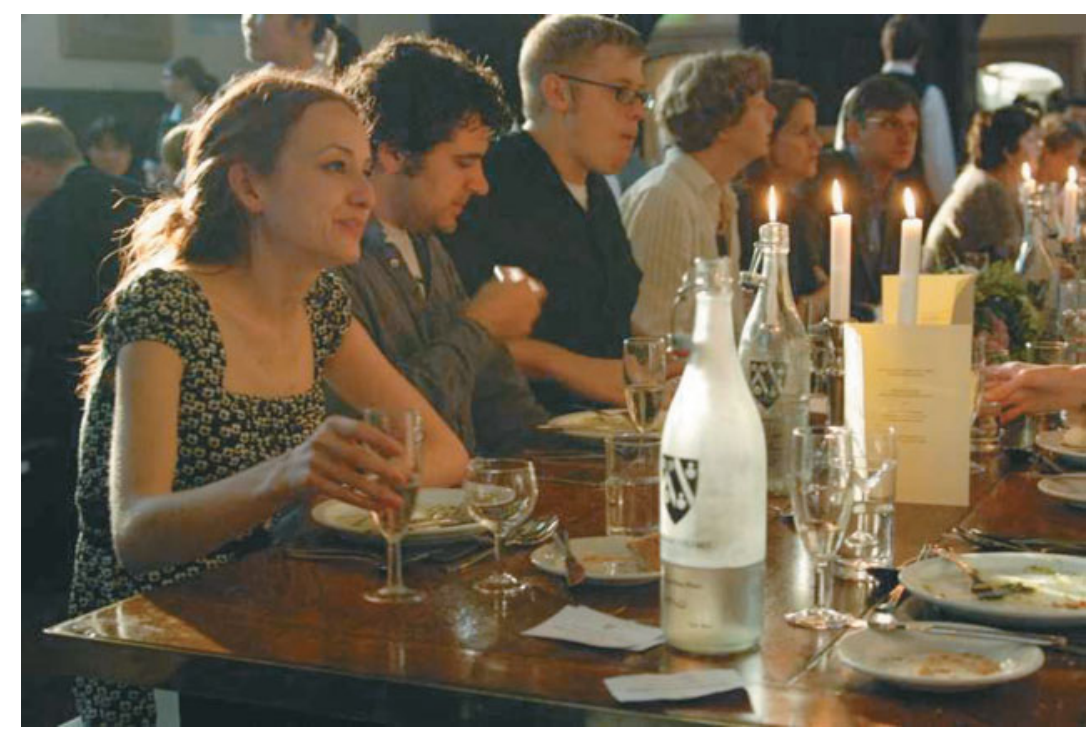

Figure 8. Patricia Sánchez-Blázquez, Philip Hopkins, Christopher Hayward, Sofia Feltzing, Katia Cunha, Verne Smith and Rosa Dominguez-Tenreiro during the symposium dinner. 